\title{
Photometric identification of the periods of the first candidate extragalactic magnetic massive stars ${ }^{\star}$
}

\author{
Yaël Nazé ${ }^{1, \star \star}$, Nolan R. Walborn ${ }^{3}$, Nidia Morrell ${ }^{2}$, Gregg A. Wade ${ }^{4}$, and Michał K. Szymański ${ }^{5}$ \\ ${ }^{1}$ Groupe d'Astrophysique des Hautes Énergies, Institut d'Astrophysique et de Géophysique, Department AGO, Université de Liège, \\ 19c Allée du 6 Août, B5c, 4000 Sart Tilman, Belgium \\ e-mail: naze@astro.ulg.ac.be \\ 2 Las Campanas Observatory, Carnegie Observatories, Casilla 601, La Serena, Chile \\ 3 Space Telescope Science Institute ${ }^{\star \star \star}, 3700$ San Martin Drive, Baltimore, MD 21218, USA \\ 4 Department of Physics, Royal Military College of Canada, PO Box 17000, Station Forces, Kingston, ON K7K 4B4, Canada \\ 5 Warsaw University Observatory, Aleje Ujazdowskie 4, 00-478 Warszawa, Poland
}

Received 12 February 2015 / Accepted 19 March 2015

\begin{abstract}
Galactic stars belonging to the Of?p category are all strongly magnetic objects exhibiting rotationally modulated spectral and photometric changes on timescales of weeks to years. Five candidate Of?p stars in the Magellanic Clouds have been discovered, notably in the context of ongoing surveys of their massive star populations. Here we describe an investigation of their photometric behaviour, revealing significant variability in all studied objects on timescales of one week to more than four years, including clearly periodic variations for three of them. Their spectral characteristics along with these photometric changes provide further support for the hypothesis that these are strongly magnetized O stars, analogous to the Of?p stars in the Galaxy.
\end{abstract}

Key words. stars: magnetic field - stars: early-type - stars: individual: AV220 - stars: individual: 2dFS 936 stars: individual: BI 57 - stars: individual: SMC159-2

\section{Introduction}

The spectral type Of?p, defined by Walborn (1972), may at first sight seem awkward with its question mark, but it is not a typo at all. The "f?p" was introduced to identify stars showing strong emission lines of $\mathrm{N}$ III $\lambda 4634-41$ and He II $\lambda 4686$ (hence the "f"), with a spectrum similar to those of supergiants, but showing other different characteristics including the unusual presence of C III $\lambda 4650$ lines in strong emission, leading to their peculiarity flag ("p"); a question mark was then added to express doubt that these stars are normal Of supergiants. Only three Galactic objects were classified in that category in the seventies: HD 108, HD 191612, and HD 148937 (Walborn 1973).

In the last decade, a growing interest in this class has appeared, as recurrent spectral variations were identified in their Balmer and He I lines. Derived periods amount to $\sim 7 \mathrm{~d}$ for HD 148937 (Nazé et al. 2008a, 2010), 538 d for HD 191612 (Walborn et al. 2004; Nazé et al. 2007; Howarth et al. 2007), and $\sim 55$ yrs for HD 108 (Nazé et al. 2001, 2006). A strong X-ray emission, brighter than that of normal $\mathrm{O}$ stars, was also found for these stars (Nazé et al. 2004, 2007, 2008a), with a modulation of the high-energy emission with the same period as the optical changes (Nazé et al. 2010). Finally, optical photometric changes (Nazé 2004; Barannikov 2007) as well as variations of line profiles in the UV range (Marcolino et al. 2012, 2013) were also reported, and they display the same properties (same

$\star$ Figures 3, 5, 7, and 8 are available in electronic form at http: //WwW . aanda.org

$\star \star$ FNRS Research Associate.

$\star \star \star$ Operated by AURA, Inc., under NASA contract NAS5-26555. period, simultaneous extrema) as the changes in the optical spectra.

After a strong magnetic field was detected in HD 191612 (Donati et al. 2006), the magnetic oblique rotator scenario was proposed to explain these peculiar properties. The monitoring of Wade et al. (2011) confirmed the proposed scenario for that star. In this model, the stellar winds of massive stars are confined in the equatorial regions by a strong dipolar magnetic field and, as the star rotates, these regions come in and out of direct view, generating the observed recurrent variations found at all wavelengths. In this context, the recurrence timescale is the rotational period, and long rotation periods may then be explained by magnetic braking over the lifetime of the star (ud-Doula et al. 2009). This implied that all Of?p stars are strongly magnetic, and HD 108 and HD 148937 were indeed soon found to be so (Hubrig et al. 2008, 2011, 2013; Martins et al. 2010; Wade et al. 2012a).

At the same time, new Of?p stars were identified in the Galaxy, with very similar characteristics (Walborn et al. 2010): NGC 1624-2 (period of $158 \mathrm{~d}$, Wade et al. 2012b) and CPD-28 2561 (Hubrig et al. 2011, 2013; period of $73 \mathrm{~d}$, Wade et al. 2015). It must be stressed that, while about ten $\mathrm{O}$ stars are now known to be magnetic, the only actual class of magnetic massive stars is the Of?p category. Spotting the typical spectral properties of Of?p stars therefore constitutes a powerful, albeit indirect, way to identify a magnetic O-type star. However, some stars with spectral types O3.5-5.5 actually display strong C III lines naturally (i.e. in the absence of a magnetic field), which led to the definition of a separate spectral type dubbed "Ofc" (Walborn et al. 2010; Martins \& Hillier 2012). The presence of strong C III lines is therefore not sufficient for classifying 
Table 1. Our targets, with their position and their star and field IDs in ASAS and OGLE databases.

\begin{tabular}{|c|c|c|c|c|c|c|}
\hline Star & RA & Dec & ASAS & OGLE-II & OGLE-III & OGLE-IV \\
\hline SMC159-2 & $00 \mathrm{~h} 49 \mathrm{~m} 58.72 \mathrm{~s}$ & $-73^{\circ} 19^{\prime} 28.4^{\prime \prime}$ & & $\begin{array}{l}\text { SMC_SC5\#95303 } \\
(\Delta=-\mathbf{0 . 0 4 2} \mathbf{~ m a g})\end{array}$ & SMC100.8\#52967 & $\begin{array}{c}\text { SMC720.27\#273 } \\
(\Delta=-\mathbf{0 . 0 5 7} \mathbf{~ m a g})\end{array}$ \\
\hline 2dFS 936 & $00 \mathrm{~h} 53 \mathrm{~m} 29.95 \mathrm{~s}$ & $-72^{\circ} 41^{\prime} 44.5^{\prime \prime}$ & $\begin{array}{c}005329-7241.6,005330-7241.6 \\
005331-7241.8,005330-7241.7 \\
\text { and } 005329-7241.4\end{array}$ & $\begin{array}{c}\text { SMC_SC6\#237339 } \\
(\Delta=-\mathbf{0 . 0 1 3} \mathbf{~ m a g})\end{array}$ & SMC101.2\#21946 & $\begin{array}{c}S M C 719.11 \# 68 \\
(\Delta=-\mathbf{0 . 0 3 2} \mathbf{~ m a g})\end{array}$ \\
\hline AV 220 & $00 \mathrm{~h} 59 \mathrm{~m} 09.97 \mathrm{~s}$ & $-72^{\circ} 05^{\prime} 48.3^{\prime \prime}$ & $\begin{array}{c}005910-7205.8 \\
\text { and } 005909-7205.8\end{array}$ & & SMC108.3\#6501 & $\begin{array}{c}\text { SMC725.16\#45 } \\
(\Delta=+\mathbf{0 . 0 0 6} \mathbf{~ m a g})\end{array}$ \\
\hline BI 57 & $05 \mathrm{~h} 01 \mathrm{~m} 08.59 \mathrm{~s}$ & $-68^{\circ} 11^{\prime} 45.1^{\prime \prime}$ & $\begin{array}{c}\text { 050107-6811.6, 050109-6811.8, } \\
\text { 050111-6811.6, 050106-6811.6, } \\
\text { and 050108-6811.8 }\end{array}$ & & LMC125.2\#9 & $\begin{array}{c}\text { LMC510.32\#25733 } \\
(\Delta=+\mathbf{0 . 0 4 0} \mathbf{~ m a g}) \\
\text { and LMC532.08\#12629 } \\
(\Delta=+\mathbf{0 . 0 2 6} \mathbf{~ m a g})\end{array}$ \\
\hline LMC164-2 & $05 \mathrm{~h} 13 \mathrm{~m} 49.88 \mathrm{~s}$ & $-69^{\circ} 23^{\prime} 21.7^{\prime \prime}$ & $\begin{array}{l}\text { 051351-6923.1, 051346-6923.1, } \\
051349-6923.4,051351-6923.1 \\
051350-6923.4,051350-6923.1\end{array}$ & $\begin{array}{c}\text { LMC_SC9\#216949 } \\
(\Delta=-\mathbf{0 . 0 0 3} \mathbf{~ m a g})\end{array}$ & LMC111.2\#10462 & $\begin{array}{l}\text { LMC503.15\#28081 } \\
(\Delta=+\mathbf{0 . 0 1 3} \mathbf{~ m a g})\end{array}$ \\
\hline
\end{tabular}

Notes. Shifts applied to OGLE-II and OGLE-IV datasets to bring them in line with OGLE-III data are also provided (in bold). $2 \mathrm{dFS} 936$ is also known under the name [MD2001] Anon 1; ASAS dataset 051346-6923.1 was discarded as it contains only two flag "A" points and in one of them the star appeared much brighter than in all other data.

a star as Of?p. Moreover, it is now known that the C III emission lines in some Of?p spectra disappear entirely at certain phases, while in others they remain strong or weak at all phases. Hence, the identification of an Of?p-type star is now rather performed through a large body of evidence: peculiar spectral characteristics (e.g. in the optical: emission lines narrower than absorption lines, emissions or P Cygni features within Balmer and He I lines, etc.) that are often periodically variable, small recurrent variations in brightness, abnormally bright X-ray emissions, and strong dipolar magnetic fields (see e.g. Nazé et al. 2008b).

Like magnetic fields, metallicity is a key ingredient for many aspects in massive stars' lives (formation, evolution, winds). It is therefore of utmost interest to study whether magnetism also occurs, and with what characteristics, at other metallicities. The closest laboratories for such tests are the Magellanic Clouds (MCs), and the best way to find likely magnetic stars is to locate Of?p stars. Indeed, other kinds of magnetic stars, e.g. active M dwarfs or Ap stars (Maitzen et al. 2001; Paunzen et al. 2011), might be easier to analyse, but the much higher intrinsic brightness of $\mathrm{O}$ stars makes them better targets in the MCs. In fact, peculiarities typical of Galactic Of?p stars have already been detected for five stars in the MCs: AV 220 (Walborn et al. 2000; Massey \& Duffy 2001), 2dFS 936 (Massey \& Duffy 2001; Evans et al. 2004), BI 57 (Howarth, priv. comm.; Walborn et al., in prep.), SMC159-2 and LMC164-2 (Massey et al. 2014). A sixth one, LMC N82 (or Brey 3a), was proposed by HeydariMalayeri \& Melnick (1992), but it was classified as Ofp by Walborn et al. (2003). Nothing more is currently known about these five stars, as their great distance makes the investigations difficult. This paper provides the first step forward, with the analysis of photometric data of these stars, with the aim of identifying a key characteristic of magnetic Of?p stars: periodic variability consistent with a long (weeks-years) rotational period. Section 2 presents the observations used to do so, while Sect. 3 presents our results, and Sect. 4 concludes this paper.

\section{Data}

In Galactic Of?p stars, periods range from a week to decades. Trying to pinpoint the recurrence timescale from random spectral observations can thus be very time-consuming. It is even more the case when the faintness of the object imposes long exposures to get spectra of good quality, as for MC objects.
However, Galactic Of?p stars do not only display spectral variations, they also show photometric changes, of the order of 0.05 mag for HD 108 and HD 191612 (Koen \& Eyer 2002; Nazé 2004; Barannikov 2007). Brightness variations are much easier to detect for MC objects since there have been several photometric surveys of these regions over the years.

We thus searched the archives of the OGLE-II (Udalski et al. 1997) ${ }^{1}$ and ASAS (Pojmanski 1997) ${ }^{2}$ projects. We found data (in the $I$-band for OGLE-II, $V$-band for ASAS) for the five candidate Of?p stars in the MCs. From these datasets, only the best quality data were kept (box "good quality" selected for OGLE-II when downloading data - we note, however, that data with flag down to F remain; only data with flag A were kept for ASAS). For OGLE-II, two photometric datasets are available: one called DIA which refers to Difference Image Analysis (Alard \& Lupton 1998; Wozniak 2000), and one called PSF which refers to PSF fitting (Szymanski 2005). The analyses were performed on both sets of magnitudes: they gave similar results. As DIA data are less noisy (and known to be more reliable, see Szymanski 2005), the numbers presented below refer to them.

Further OGLE data, from OGLE-III and OGLE-IV campaigns, were also available. The third phase of the OGLE project (OGLE-III, Udalski 2003) was realized during the years 2001-2009 using an 8-chip CCD mosaic. The photometry was obtained using the DIA method. OGLE-IV is the currently ongoing, fourth phase of the project. It started in 2010, using a huge, 32-chip CCD mosaic and the DIA reduction scheme. Analysing the OGLE datasets we noted small residual magnitude shifts between the three campaigns. To correct for them, we computed, for each dataset, the mean of all data, the median of the $10 \%$ largest data (i.e. 90th percentile), and the median of the $10 \%$ smallest data (i.e. 10th percentile). OGLE-III data are considered as the best calibrated amongst the OGLE collaboration, and were then used as reference: the differences between the derived mean and median values of OGLE-II/IV and their equivalent in OGLE-III data yield shifts that were applied to the OGLE-II/IV before analysis. We note that using means or any of the medians yields the same shifts. For reference, they are shown in Table 1 along with the IDs of the fields and stars in the different programmes.

\footnotetext{
1 http://ogle.astrouw.edu.pl/

2 http://www.astrouw.edu.pl/asas/
} 
Although it is currently off-line, we managed to get access to the EROS-2 database (see Tisserand et al. 2007 for details). Data are available for three of our stars (AV 220, 2dFS 936, and LMC164-2). They cover a shorter time interval than OGLE data, from HJD 2450300 to 2452700 , but can be used as a confirmation tool. Other photometric surveys have no available data for these objects (e.g. Catalina sky survey) or have no public data.

On each of these datasets, we first applied a $\chi^{2}$ test for constancy and then several period search algorithms. They fall in four categories: (1) the Fourier algorithm adapted to sparse/uneven datasets (Heck et al. 1985; Gosset et al. 2001; a method rediscovered recently by Zechmeister \& Kürster 2009 - these papers also note that the method of Scargle 1982, while popular, is not fully correct, statistically); (2) two different string length methods (Lafler \& Kinman 1965; Renson 1978); (3) three binned analyses of variances (Whittaker \& Robinson 1944; Jurkevich 1971 which is identical, with no bin overlap, to the "pdm" method of Stellingwerf 1978; and Cuypers 1987 - which is identical to the "AOV" method of SchwarzenbergCzerny 1989); and (4) conditional entropy (Cincotta et al. 1999; Cincotta 1999, see also Graham et al. 2013). Each of these methods has its advantages and its drawbacks - the most reliable being the Fourier method while the fastest ones usually are analyses of variances - but a period clearly identified by all of them is certainly real. All uncertainties reported in this paper correspond to $1 \sigma$ errors.

In view of the results (see next section), additional spectra are needed. Indeed, usually, only one spectrum for each object exists in the literature, but more than one spectrum is needed to examine variations. Spectral monitorings of AV 220, BI 57, and 2dFS 936 are examined in a companion paper (Walborn et al., in prep.). For the remaining two objects, the published spectra of LMC164-2 and SMC159-2 were obtained in the framework of a WR survey in the MCs (Massey et al. 2014). This campaign is not completed yet, which enabled us to get one additional spectrum of SMC159-2 in September 2014. As it is the sole new one, it will be presented here rather than by Walborn et al. (in prep.) who present larger spectral sets. As with the two previous spectra (Massey et al. 2014), this new spectrum was obtained at Las Campanas Observatory with the 6.5-m Magellan II (Clay) telescope using the MagE spectrograph (Marshall et al. 2008) and a $1^{\prime \prime}$ slit which provided a resolution ranging from 0.7 to $1.7 \AA$ going from the blue to the red end of the optical spectrum. The exposure time was $3 \times 600 \mathrm{~s}$. The data were processed with a combination of the IRAF mtools package, originally developed by Jack Baldwin for the reduction of MIKE data, and standard IRAF échelle tasks as described by Massey et al. (2012). ThAr lamps were used to derive wavelength solutions, and flux standards were observed on each night in order to flux calibrate the data. The two spectra of SMC159-2 were normalized considering clean continuum windows.

\section{Results}

Before looking at each object in turn, a note about the data quality should be made. We expect the photometric variations to be very small (in the Galaxy, variations no larger than 0.05 mag are seen, see previous section). OGLE data typically have errors of 0.003-0.004 mag while the errors on ASAS and EROS-2 data are ten times larger (0.04-0.05 mag for ASAS and 0.015-0.15 mag with a median of $0.03 \mathrm{mag}$ for EROS-2). In addition, the extraction apertures are different (from about $1.5^{\prime \prime}$ in OGLE to a radius of $15^{\prime \prime}$ in ASAS, with EROS-2 in-between), leading to different degrees of contamination. It is therefore unsurprising
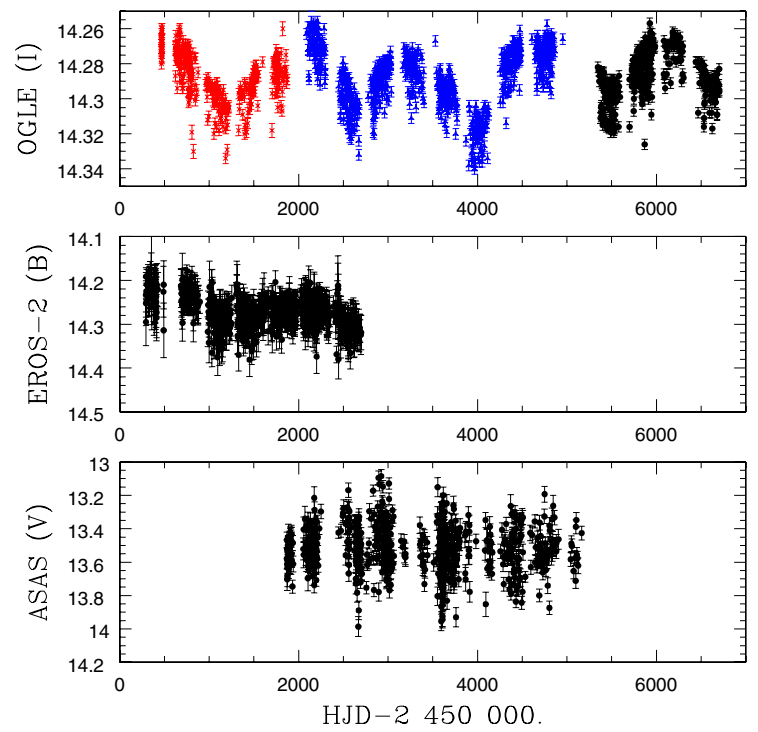

Fig. 1. Photometric variations of $2 \mathrm{dFS} 936$, observed in the $I$-band (OGLE, top - OGLE-II data are shown by red crosses, OGLE-III by blue triangles, and OGLE-IV by black dots), non-standard $B$-band (EROS-2, middle), and $V$-band (ASAS, bottom). It must be noted that the top panel spans only 0.10 mag while the middle and bottom panels span much larger ranges ( 0.4 mag and $1.2 \mathrm{mag}$, respectively).

that the different datasets do not produce exactly the same results. A good example is provided with 2dFS 936: the coherent variations detected by OGLE have an amplitude of $0.015 \mathrm{mag}$ and this is confirmed in EROS-2 data (see below), but this signal is buried in ASAS noise as can be seen from a direct comparison of data taken by the two surveys at similar epochs (Fig. 1). Therefore, we definitely trust more OGLE data than ASAS data.

\section{1. $A V 220$}

For AV 220, the photometric data show a dispersion that is significantly larger than the error bars (e.g. Fig. 2, left panel), and are thus found significantly variable in a $\chi^{2}$ test. However, period search algorithms do not find a clear and unequivocal signal (Fig. 2, middle panel). With the ASAS dataset, most methods display some low-amplitude peaks around 10-15d, but periodograms look overall like white noise. For OGLE data, power is found only at the lowest frequencies, but different methods disagree on the value of the best period $(1299 \mathrm{~d}, 3846 \mathrm{~d}$ ?), and prewhitening with these frequencies does not yield a flat periodogram (Fig. 2, right panel). This may come from a changing variation timescale, with a variation apparently more rapid in the OGLE-IV dataset. Indeed, when looking at photometry over time (Fig. 2, left panel), brightness maxima are seen around HJD $=2452900,2455200$, and 2456600 while minima are seen around HJD $=2452000,2454400$, and 2456000 (we note, however, that 2455200 and 2456600 are at the beginning and end, respectively, of the dataset). To assess this difference, we perform period searches on the OGLE-III and OGLE-IV datasets separately: periodograms based on OGLE-III data favour "periods" of $2500 \mathrm{~d}$ or $3700 \mathrm{~d}$, while those calculated with OGLE-IV data yield shorter values of 1200-1400d. The change with time is thus not an effect of the annual gaps, but a true variation. The EROS-2 data produce similar results and could not provide a stronger constraint on the timescales. A secure period has thus not yet been identified for AV 220, and further monitoring is needed to better understand the photometric variations of this star. 

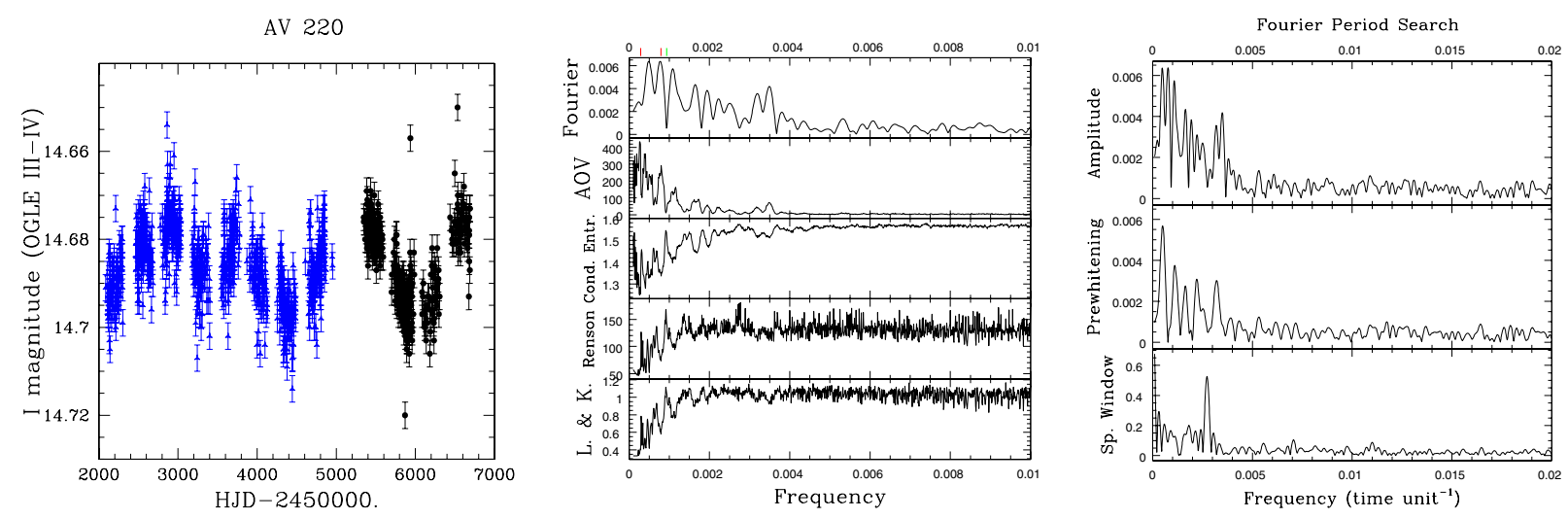

Fig. 2. Left: OGLE-III and IV lightcurve for AV 220 (colour scheme as in Fig. 1). Middle: comparison of the different period search methods, with tickmarks at the top indicating periods of $3846 \mathrm{~d}, 1299 \mathrm{~d}$, and $1100 \mathrm{~d}$. The presence of a signal is marked by a maximum for Fourier and AOV methods, but by a minimum for conditional entropy, Renson and Lafler \& Kinman methods. Right: Fourier periodogram for the raw (top) and prewhitened (middle, for the best-fit Fourier period $P=1299$ d) data, along with the spectral window (bottom).

BI57

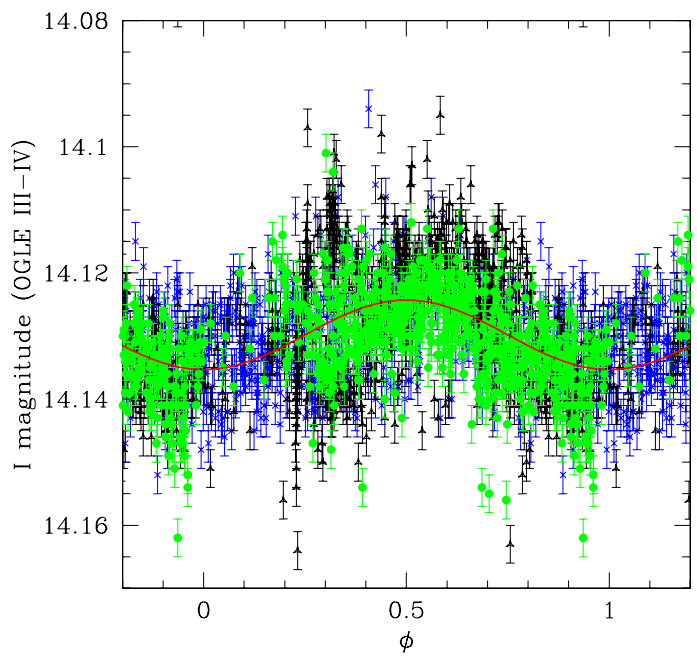

Fig. 4. Photometric variations of BI 57, phased using the best-fit Fourier ephemeris (period of $400 \mathrm{~d}$, see Table 2). The thick red line corresponds to the best-fit Fourier sinusoid for that period, while the symbols for data are as in Fig. 3.

\section{2. $B 157$}

Regarding BI 57, it must be noted that, in the LMC532.08 field, the star is located close to the outer edge of the mosaic, where the template image is of worse quality and the photometry calibration less reliable owing to vignetting. However, discarding this dataset from the analysis does not change significantly the following results. BI 57 displays obvious and significant variability of its photometry (Fig. 3, left panel). In period searches, ASAS data yield a marginal detection at a Fourier period of $P=96.7 \pm 0.3 \mathrm{~d}$, while OGLE data clearly favour a period of $400 \pm 3 \mathrm{~d}$ (Fourier) or its double $(787 \pm 14 \mathrm{~d}$, entropy \& variance analyses). While the photometry appears rather well phased with the latter periods (e.g. Fig. 4), it must be noted that the dispersion in the phased diagram appears higher than for the three following Of?p candidates, pointing to incompatibilities of the OGLE dataset with a strict periodicity. As further evidence, a model considering the sum of two sinusoids with periods $400 \mathrm{~d}$ and $787 \mathrm{~d}$ does reproduce the average magnitudes of each observing run, but sometimes fails to reproduce the detailed trends (increase/decrease) of the observed variations (see such a model
Table 2. Properties of the detected periods, from the Fourier method.

\begin{tabular}{lccc}
\hline \hline ID & $T_{0}-2450000$ & Period $(\mathrm{d})$ & Amplitude $(\mathrm{mag})$ \\
\hline SMC159-2 & $3598.706 \pm 0.049$ & $14.914 \pm 0.004$ & $0.00719 \pm 0.00015$ \\
2dFS 936 & $3993.3 \pm 1.7$ & $1370 \pm 30$ & $0.01504 \pm 0.00011$ \\
LMC164-2 $^{\dagger}$ & $4027.338 \pm 0.015$ & $7.9606 \pm 0.0010$ & $0.00926 \pm 0.00011$ \\
BI 57* $^{*}$ & $5302.2 \pm 1.2$ & $400.0 \pm 3.5$ & $0.00550 \pm 0.00010$ \\
\hline
\end{tabular}

Notes. $T_{0}$ (hence $\phi=0$ ) corresponds to minimum brightness; ${ }^{(*)}$ not strictly periodic (see text); $\left.{ }^{\dagger}\right)$ there are additional periodicities in 2dFS 936 (see text).

in red in the left panel of Fig. 3). Furthermore, prewhitening by these periods does not yield a flat periodogram as it should in the case of a strict periodicity (e.g. Fig. 3, right panel). Analysing the three OGLE datasets separately confirms the presence of differences: for example, the $787 \mathrm{~d}$ peak is higher in the Fourier periodogram of OGLE-III data while the periodograms based on the two OGLE-IV datasets favour the $400 \mathrm{~d}$ peak. Therefore, while it is clear that some changes occur with periods of $400 \mathrm{~d}$ and $787 \mathrm{~d}$, such periodicities cannot explain the full range of variations in BI 57.

\section{3. $2 d F S 936$}

The case of 2dFS 936 is very different. The data appear significantly variable (the star was in fact reported as an "irregular variable" by Kourniotis et al. 2014); a clear long-term modulation is readily detected by eye in OGLE data (Fig. 1). It is indeed detected independently by the different period search methods (Fig. 5, left panel), with a best-fit period of $1370 \pm 30 \mathrm{~d}$ (Table 2). Prewhitening by this period yields a flat periodogram while phased photometry results in very coherent variations (Fig. 5, middle and right panels), securing that detection. In addition, the spectroscopic changes of $2 \mathrm{dFS} 936$ seem to occur in phase with the same period (see Walborn et al., in prep.): this $1370 \mathrm{~d}$ period clearly appears as the rotation period of a typical Of?p star.

It should be noted, however, that the maximum at HJD 2453250 and the following minimum at HJD $\sim 2454000$ occur when the star was fainter than for other maxima and minima. Examining further the periodogram, we found that a second set of peaks exists, along with their daily aliases: in the interval $0 .-4.5 \mathrm{~d}^{-1}$, their frequencies are $0.48525 \mathrm{~d}^{-1}$ and $0.51246 \mathrm{~d}^{-1}$, 
2dFS $936(P=1 / 2.48952 d)$

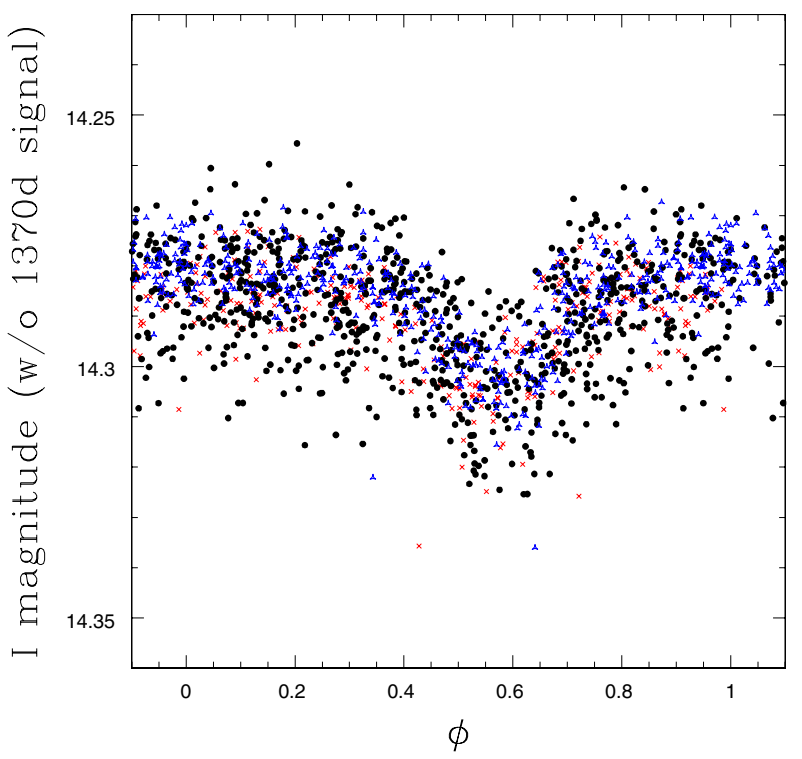

Fig. 6. Photometric changes of $2 \mathrm{dFS} 936$, once the $1370 \mathrm{~d}$ variation is removed. This plot uses the $T_{0}$ of Table 2 and a period of $0.402 \mathrm{~d}$ (i.e. a frequency of $2.48952 \mathrm{~d}^{-1}$ ). The symbols for data are as in Fig. 1 .

$1.48676 \mathrm{~d}^{-1}, 2.48952 \mathrm{~d}^{-1}, 3.48952 \mathrm{~d}^{-1}$ and $3.49224 \mathrm{~d}^{-1}$, and $4.49228 \mathrm{~d}^{-1}$. With the current dataset, it is difficult to assess which frequency is the correct one: data points prewhitened for the $1370 \mathrm{~d}$ variation yield nicely phased variations for all frequencies larger than $1 \mathrm{~d}^{-1}$ (e.g. Fig. 6). The origin of this additional variation may not be linked to the Of?p phenomenon, it may rather be related to pulsations or to eclipses in a binary system. In the latter case, the orbital period would then be the double of one of the periods detected by period searches and the lightcurve with two similar eclipses would indicate similar stars. However, such a period $(0.4-4 \mathrm{~d})$ would be very short for such massive objects and a line-of-sight coincidence between $2 \mathrm{dFS} 936$ and an eclipsing binary could then be required. Identifying the correct frequency and understanding its nature implies reobserving 2dFS 936, this time over several nights with a high sampling frequency to avoid any ambiguities.

Formally, using a $\chi^{2}$ test, the EROS-2 data in their blue band (where most data points are available) do not deviate significantly from a constant, but period search methods, being very sensitive to periodic signals, readily confirm the signals found in OGLE data: main period at $1389 \pm 80 \mathrm{~d}$ with an amplitude in $B$-band of $0.0206 \pm 0.0013 \mathrm{mag}$, secondary period at $2.05 \mathrm{~d}$ (or its aliases).

\section{4. $L M C 164-2$}

A $\chi^{2}$ test shows that LMC164-2 is significantly variable in both ASAS and OGLE datasets. All period searches on OGLE data reveal a very clear peak at $P=7.96 \mathrm{~d}$ : values range from $7.9598 \mathrm{~d}$ for variance methods to $7.9612 \mathrm{~d}$ for Renson's method, which is fully consistent with the $0.0010 \mathrm{~d} 1 \sigma$ error (see Table 2 and left panel of Fig. 7). Moreover, this period yields coherent variations with phase and, when eliminated from the data, leaves a flat periodogram (Fig. 7, middle and right panels). The EROS-2 data in their blue band (where most data points are available) of LMC164-2 have the lowest errors (always <0.045 mag). Formally, they do not deviate significantly from a constant, but period search methods readily confirm the signal found in OGLE data, with a period of $7.959 \pm 0.003 \mathrm{~d}$ and an amplitude in this band of $0.0089 \pm 0.0016 \mathrm{mag}$. Periodograms and phased variations are very similar to those shown in Fig. 7. This signal is thus undoubtly real, though it remains buried in ASAS noise.

\subsection{SMC159-2}

SMC159-2 appears only in the OGLE database and the OGLE data appear significantly variable, as shown by a $\chi^{2}$ test. Period searches on OGLE data reveal a very clear peak at $P=14.91 \mathrm{~d}$ : values range from $14.912 \mathrm{~d}$ for variance methods to $14.915 \mathrm{~d}$ for the conditional entropy method, a difference which is well within the $0.004 \mathrm{~d} 1 \sigma$ error (see Table 2 and Fig. 8). This period yields coherent phased variations and, when eliminated from the data, leaves a flat periodogram (Fig. 8). Again, this leaves little doubt about the reality of the signal.

Two spectra of SMC159-2 are available, one taken in 2013 (on HJD $=2456640.551)$ and one in 2014 (on HJD = $2456903.691)$. Using the best-fit ephemeris derived from Fourier algorithm (Table 2), we found that the 2013 and 2014 spectra of SMC159-2 were acquired at two very different phases, 0.96 and 0.60 , respectively. These spectra display many features that are typical of Galactic Of?p stars (Fig. 9). The He II absorption lines remain essentially constant, indicative of a predominantly photospheric origin, and they are broader than He I absorption lines, as typically observed in Of?p spectra. We note that the apparent spectral type does not change much, the He II $\lambda 4541 / \mathrm{He}$ I $\lambda 4471$ ratio remaining consistent with a spectral type of O6. In addition, the emission lines appear relatively narrow (e.g. FWHM of $\sim 280 \mathrm{~km} \mathrm{~s}^{-1}$ for $\mathrm{H} \alpha$ ), i.e. much narrower that typical wind $\mathrm{H} \alpha$ emission of O-stars, as is usually observed in Of?p stars. In parallel, many variations typical of Of?p stars are also observed. The Balmer lines and He II $\lambda 4686$ emission lines are stronger in 2014, although with one exception (HD 148937) the differences are generally smaller than in the spectra of Galactic Of?p stars. These lines appear slightly redshifted at maximum, as is seen e.g. in CPD-28 2561 (Wade et al. 2015). In addition, the N III and C III emission lines near 4630-4650 $\AA$ are stronger in 2014, the C III emissions nearly disappearing in 2013. The most extreme variation occurs in the metastable He I $\lambda 5876$ line, which changes from an apparent inverse P Cygni profile at minimum, to a much stronger double emission line at maximum, the second peak appearing longward of the single minimum emission. This could be related to the redshifts observed for other emission lines. If this star is confirmed to be magnetic, then the physical origins of these diverse behaviours are linked to the geometry of the rotating magnetospheric structure. A full spectroscopic monitoring will thus be needed to derive the stellar properties, as was done e.g. for HD 191612 (Wade et al. 2011).

In the Galaxy, Of?p stars brighten when their emission increases (Walborn et al. 2004; Barannikov 2007). The phase of the 2013 spectrum of SMC159-2 $(\phi=0.96)$ corresponds to a brightness minimum, while the phase of the 2014 spectrum $(\phi=0.60)$ indicates that it was taken close to brightness maximum. Furthermore, a direct comparison is possible as the last photometric data were acquired close in time to the 2013 spectrum: the $I$-magnitude was $15.544 \pm 0.003$ on HJD $=2456639.571$, i.e. the star was indeed faint at the time. There is thus a direct correlation between the strength of the emission lines and the brightness of the star: SMC159-2 behaves in a manner similar to the Galactic Of?p stars. 


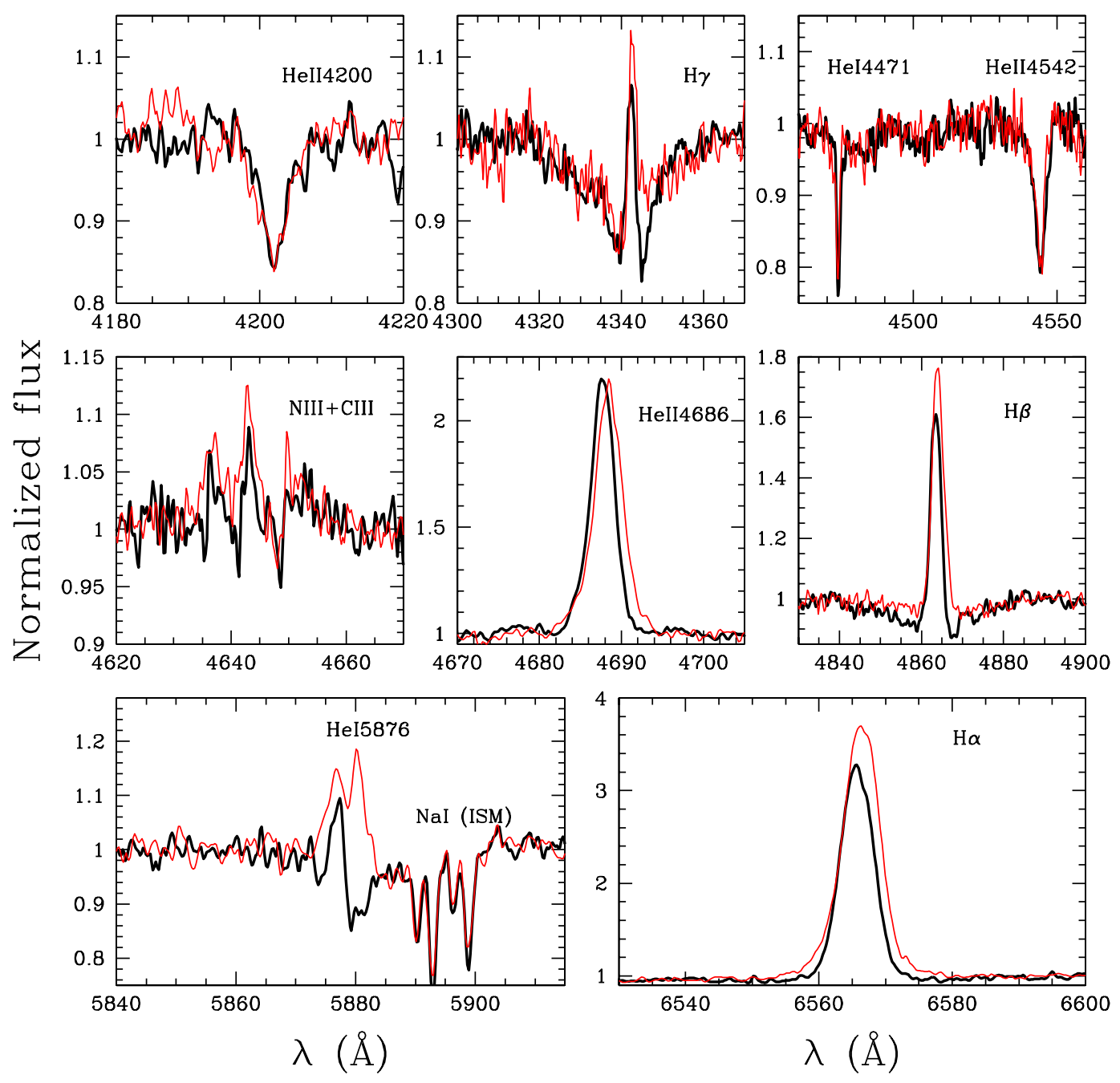

Fig. 9. Spectra of SMC159-2 recorded in 2013 ( $\phi=0.96$, thick black line) and $2014(\phi=0.60$, thinner red line) in selected spectral windows: clear changes in Balmer and He I lines can be seen, with stronger emissions in 2014, while photospheric He II absorptions and interstellar lines arising in both the Galaxy and the SMC (e.g. Na D lines near $5900 \AA$ ) remain constant.

Equivalent widths (EWs) of $\mathrm{H} \alpha$ and $\mathrm{He}$ II $\lambda 4686$ were evaluated by integrating the spectra in the $6550-6600 \AA$ and $4680-4697 \AA$ intervals, respectively, as well as by fitting gaussians to the emission peaks. Derived EWs amount to $13.7 \pm 0.5 \AA$ and $4.3 \pm 0.2 \AA$ in 2013 and to $19.2 \pm 0.2 \AA$ and $5.0 \pm 0.2 \AA$ in 2014 for $\mathrm{H} \alpha$ and $\mathrm{He}$ II $\lambda$ 4686, respectively. It must be noted that there is no nebulosity near SMC159-2, hence the recorded Balmer and He I lines are uncontaminated: the emissions and their variability are truly linked to the star. We also note that the emission in $\mathrm{H} \alpha$ is very strong, suggesting a large quantity of emitting material. Amongst magnetic $\mathrm{O}$ stars, the peak EW of $\mathrm{H} \alpha$ emission generally amounts to a few Ångströms (e.g. Howarth et al. 2007), except for the exceptional case of the highly magnetized NGC 1624-2 which presents a peak EW of about $27 \AA$ (Wade et al. 2012b). Figure 10 shows the peak EW of the $\mathrm{H} \alpha$ line for $\theta^{1}$ Ori $\mathrm{C}$ and the five Galactic Of?p stars as a function of their surface magnetic field strengths $B_{d}$. This figure demonstrates the expected increase in emission with the field strength, and hence with the magnetospheric volume. For slow rotators such as Of?p stars, this magnetospheric volume is determined by the Alfvén radius: it is thus a function of both the magnetic field strength and wind momentum, but the latter was assumed to be approximately uniform for all stars in Fig. 10.

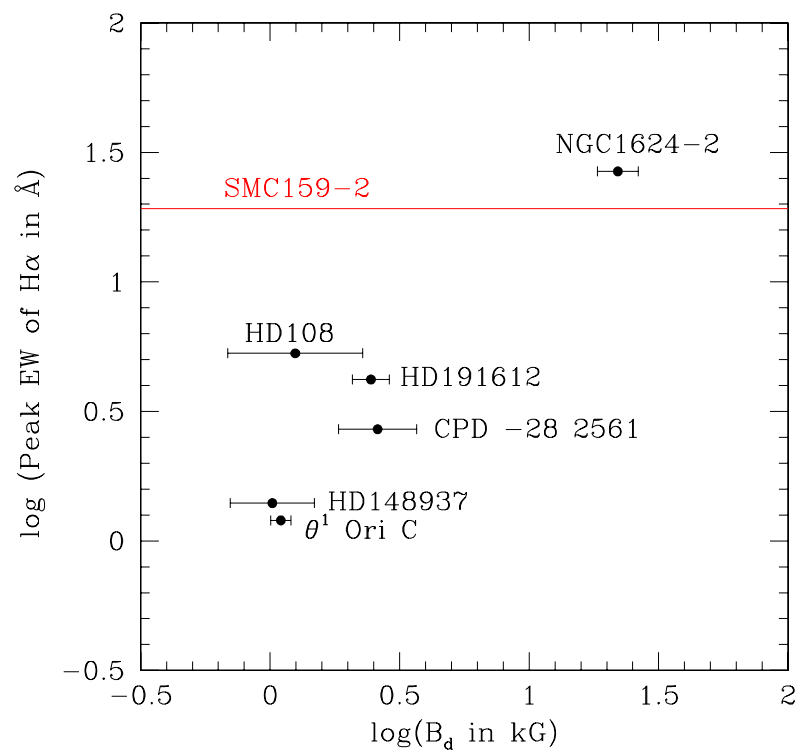

Fig. 10. Comparison of the peak $\mathrm{H} \alpha \mathrm{EW}$ and the dipolar field strength of magnetic $\mathrm{O}$ stars (using unpublished spectropolarimetry obtained by GAW for NGC 1624-2). The horizontal red line indicate the peak $\mathrm{H} \alpha$ EW of SMC159-2. 
A forthcoming paper (Wade et al., in prep.) will examine in more detail the theoretical expectations for the MC Of?p stars. Based on this figure and the outstanding strength of its $\mathrm{H} \alpha$ emission, we can predict that the magnetic field of SMC159-2 is very strong, second only to that of NGC 1624-2 and likely in the range $5-15 \mathrm{kG}$.

\section{Conclusions}

The Of?p category comprises magnetic massive stars, where an oblique magnetic field provokes rotationally-modulated photometric and spectral variations in the optical, UV and X-ray domains. Up to now, such objects were only studied in detail in the Galaxy, but five candidate extragalactic Of?p stars have been proposed. No spectropolarimetry of these objects is yet available, and further spectroscopic data, though somewhat scarce, will be soon presented by Walborn et al. (in prep.).

In this paper, we analysed the photometry of these five objects. Three of them behave exactly like magnetic Of?p stars in the Galaxy: the photometric amplitudes, the roughly sinusoidal form of the variations, and the strict and stable periodicity with periods from $8 \mathrm{~d}$ to $1370 \mathrm{~d}$ are similar to what is found in Galactic cases. These stars thus have a high probability of being magnetic, though it is difficult to derive the system's geometry and magnetic field strength with the photometric data alone. Moreover, simultaneous spectral variations observed in SMC159-2 are also reminiscent of the behaviour of Galactic Of?p stars. It can be noted that the $\mathrm{H} \alpha$ line of SMC159-2 is remarkably strong. This leads us to predict that its magnetosphere is very large and that its magnetic field is especially strong, likely only second to that of the outstanding star NGC 1624-2.

For the remaining two Of?p candidates, significant photometric variability is observed, but strict periodicity cannot be ascertained. Their unexplained photometric variations may well be a consequence of multiple variability origins (e.g. rotation in combination with binarity or pulsation). Therefore, while these stars demonstrate the spectral peculiarities expected of hot magnetic Of?p stars, we are not yet able to confirm photometric variability consistent with stable, long-term rotational modulation.

This study paves the way for further observations, opening the door to the study of magnetic field and stellar wind interactions in extragalactic environment at lower metallicities.

Acknowledgements. We warmly thank Phil Massey for having uncovered three of the Of?p candidates and for sharing data as well as observing time with the authors. Y.N. thanks E. Gosset for useful discussion and acknowledges support from the Fonds National de la Recherche Scientifique (Belgium), the PRODEX XMM contracts, and the ARC (Concerted Research Action) grant financed by the Federation Wallonia-Brussels. G.A.W. acknowledge Discovery Grant support from the Natural Science and Engineering Research Council of Canada (NSERC). M.K. Szymanski acknowledges funding of the OGLE project from the European Research Council under the European Community's Seventh Framework Programme (FP7/2007-2013)/ERC grant agreement no. 246678. This work also made use of EROS-2 data, which were kindly provided by the EROS collaboration, thanks to J.B. Marquette. The EROS (Expérience pour la Recherche d'Objets Sombres) project was funded by the CEA and the IN2P3 and INSU CNRS institutes. ADS and CDS were used for preparing this document.

\section{References}

Alard, C., \& Lupton, R. H. 1998, ApJ, 503, 325

Barannikov, A. A. 2007, IBVS, 5756, 1

Cincotta, P. M. 1999, MNRAS, 307, 941
Cincotta, P. M., Helmi, A., Mendez, M., Nunez, J. A., \& Vucetich, H. 1999, MNRAS, 302, 582

Cuypers, J. 1987, A\&AS, 69, 445

Donati, J.-F., Howarth, I.D., Bouret, J.-C., et al. 2006, MNRAS, 365, L6

Evans, C. J., Howarth, I. D., Irwin, M. J., Burnley, A. W., \& Harries, T. J. 2004, MNRAS, 353, 601

Gosset, E., Royer, P., Rauw, G., Manfroid, J., \& Vreux, J.-M. 2001, MNRAS, 327,435

Graham, M. J., Drake, A. J., Djorgovski, S. G., Mahabal, A. A., \& Donalek, C. 2013, MNRAS, 434, 2629

Heck, A., Manfroid, J., \& Mersch, G. 1985, A\&AS, 59, 63

Heydari-Malayeri, M., \& Melnick, J. 1992, A\&A, 258, L13

Howarth, I. D., Walborn, N. R., Lennon, D. J., et al. 2007, MNRAS, 381, 433

Hubrig, S., Schöller, M., Schnerr, R. S., et al. 2008, A\&A, 490, 793

Hubrig, S., Schöller, M., Kharchenko, N. V., et al. 2011, A\&A, 528, A151

Hubrig, S., Schöller, M., Ilyin, I., et al. 2013, A\&A, 551, A33

Jurkevich, I. 1971, Ap\&SS, 13, 154

Koen, C., \& Eyer, L. 2002, MNRAS, 331, 45

Kourniotis, M., Bonanos, A. Z., Soszyński, I., et al. 2014, A\&A, 562, A125

Lafler, J., \& Kinman, T. D. 1965, ApJS, 11, 216

Maitzen, H. M., Paunzen, E., \& Pintado, O. I. 2001, A\&A, 371, L5

Marcolino, W. L. F., Bouret, J.-C., Walborn, N. R., et al. 2012, MNRAS, 422 2314

Marcolino, W. L. F., Bouret, J.-C., Sundqvist, J. O., et al. 2013, MNRAS, 431, 2253

Marshall, J. L., Burles, S., Thompson, I. B. et al. 2008, Proc. SPIE, 7014, 701454

Martins, F., Donati, J.-F., Marcolino, W. L. F., et al. 2010, MNRAS, 407, 1423

Martins, F., \& Hillier, D. J. 2012, A\&A, 545, A95

Massey, P., \& Duffy, A. S. 2001, ApJ, 550, 713

Massey, P., Morrell, N.I., Neugent, K.F., et al. 2012, ApJ, 748, 96

Massey, P., Neugent, K. F., Morrell, N., \& Hillier, D. J. 2014, ApJ, 788, 83

Nazé, Y. 2004, Ph.D. Thesis, Université de Liège

Nazé, Y., Vreux, J.-M., \& Rauw, G. 2001, A\&A, 372, 195

Nazé, Y., Rauw, G., Vreux, J.-M., \& De Becker, M. 2004, A\&A, 417, 667

Nazé, Y., Barbieri, C., Segafredo, A., Rauw, G., \& De Becker, M. 2006, IBVS 5693, 1

Nazé, Y., Rauw, G., Pollock, A. M. T., Walborn, N. R., \& Howarth, I. D. 2007, MNRAS, 375, 145

Nazé, Y., Walborn, N. R., Rauw, G., et al. 2008a, AJ, 135, 1946

Nazé, Y., Walborn, N. R., \& Martins, F. 2008b, Rev. Mex. Astron. Astrofis., 44, 331

Nazé, Y., ud-Doula, A., Spano, M., et al. 2010, A\&A, 520, A59

Paunzen, E., Netopil, M., \& Bord, D. J. 2011, MNRAS, 411, 260

Pojmanski, G. 1997, Acta Astron., 47, 467

Renson, P. 1978, A\&A, 63, 125

Scargle, J. D. 1982, ApJ, 263, 835

Schwarzenberg-Czerny, A. 1989, MNRAS, 241, 153

Stellingwerf, R. F. 1978, ApJ, 224, 953

Szymanski, M. K. 2005, Acta Astron., 55, 43

Tisserand, P., Le Guillou, L., Afonso, C., et al. 2007, A\&A, 469, 387

Udalski, A. 2003, Acta Astron., 53, 291

Udalski, A., Kubiak, M., \& Szymanski, M. 1997, Acta Astron., 47, 319

ud-Doula, A., Owocki, S. P., \& Townsend, R. H. D. 2009, MNRAS, 392, 1022

Wade, G. A., Howarth, I. D., Townsend, R. H. D., et al. 2011, MNRAS, 416, 3160

Wade, G. A., Grunhut, J., Gräfener, G., et al. 2012a, MNRAS, 419, 2459

Wade, G. A., Maíz Apellániz, J., Martins, F., et al. 2012b, MNRAS, 425, 1278

Wade, G. A., Barbá, R. H., Grunhut, J., et al. 2015, MNRAS, 447, 2551

Walborn, N. R. 1972, AJ, 77, 312

Walborn N. R. 1973, AJ, 78, 1067

Walborn, N. R., Lennon, D. J., Heap, S. R., et al. 2000, PASP, 112, 1243

Walborn, N. R., Howarth, I. D., Herrero, A., \& Lennon, D. J. 2003, ApJ, 588, 1025

Walborn, N.R., Howarth, I.D., Rauw, G., et al. 2004, ApJ, 617, L61

Walborn, N. R., Sota, A., Maíz Apellániz, J., et al. 2010, ApJ, 711, L143

Whittaker, E. T., \& Robinson, G. 1944, The calculus of observations; a treatise on numerical mathematics (London: Blackie)

Wozniak, P. R. 2000, Acta Astron., 50, 421

Zechmeister, M., \& Kürster, M. 2009, A\&A, 496, 577

Pages 8 to 9 are available in the electronic edition of the journal at http: //www . aanda. org 
BI 57

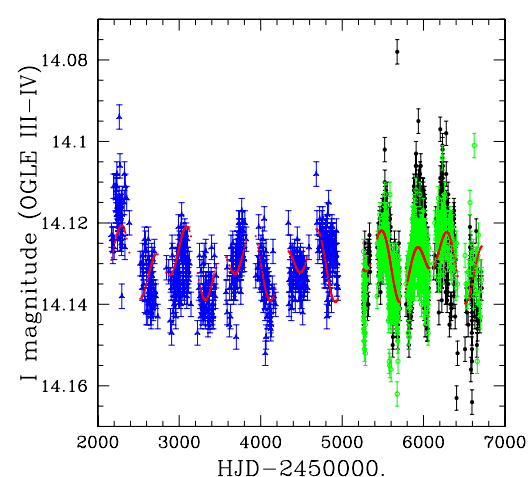

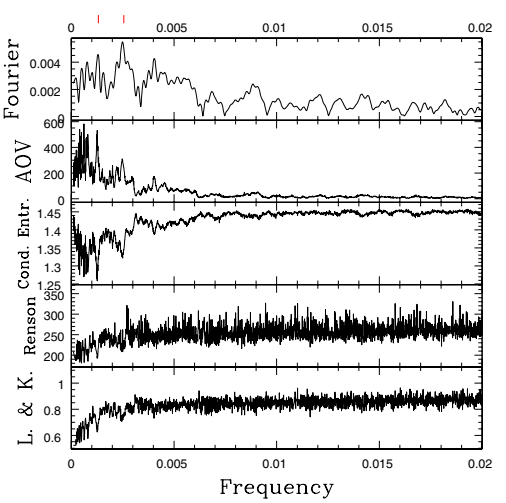

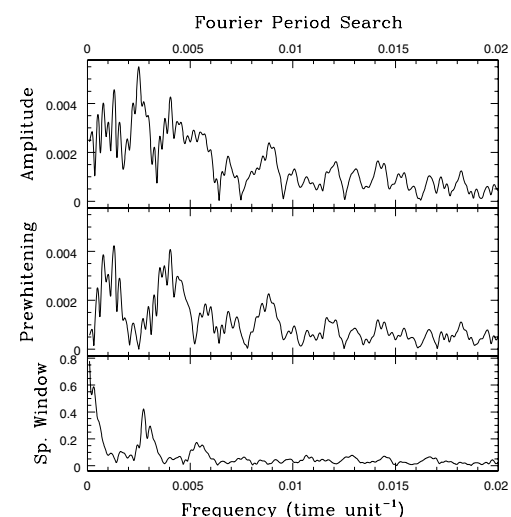

Fig. 3. Same as Fig. 2 for BI 57 (a best-fitting double sinusoid, with the two periods of $787 \mathrm{~d}$ and $400 \mathrm{~d}$ is added in red in the left panel; these periods are indicated by tickmarks in the middle panel; prewhitening in the right panel was performed for $P=400 \mathrm{~d}$ ). The potentially problematic OGLE-IV dataset (LMC532.08) is shown in green.
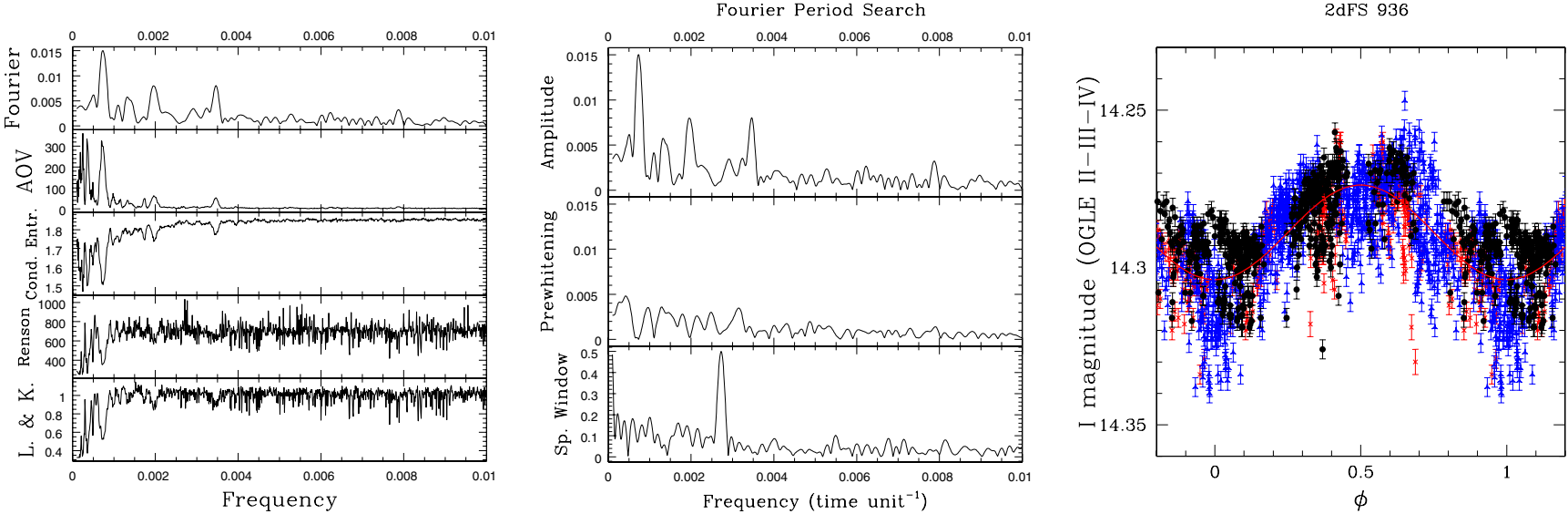

Fig. 5. Period search results for $2 \mathrm{dFS} 936$. Left: comparison of the different period search methods. The presence of a signal is marked by a maximum for Fourier and AOV methods, but a minimum for conditional entropy, Renson and Lafler \& Kinman methods. Middle: Fourier periodogram for the raw (top) and prewhitened (middle, for the best-fit Fourier period) data of 2dFS 936, along with the spectral window (bottom). Right: photometric variations of 2dFS 936, phased using the best-fit ephemeris (see Table 2). The thick red line corresponds to the best-fit Fourier sinusoid, while the symbols for data are as in Fig. 1.
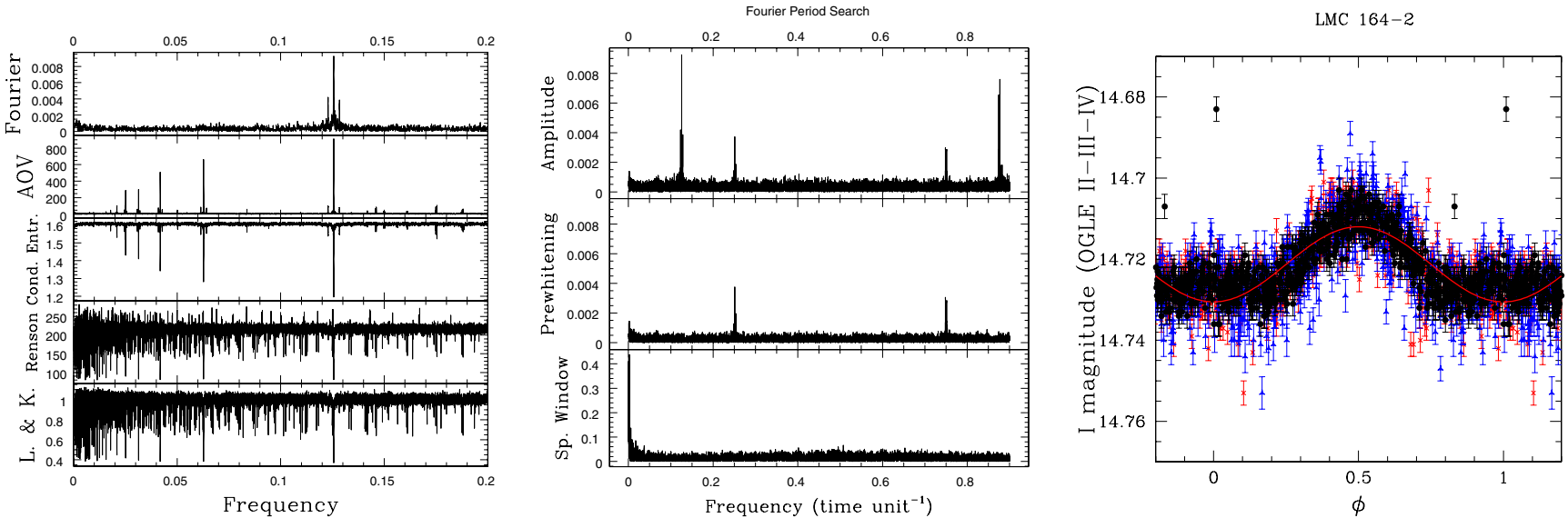

Fig. 7. Same as Fig. 5 for LMC164-2 (see Table 2 for ephemeris). We note in the left panel the presence of peaks at frequencies corresponding to multiples of the fundamental period for all methods except Fourier, and in the middle panel the best-fit signal at $0.126 \mathrm{~d}^{-1}$, its second harmonic at $0.25 \mathrm{~d}^{-1}$, and their daily aliases at $0.87 \mathrm{~d}^{-1}$ and $0.75 \mathrm{~d}^{-1}$, respectively. 
Y. Nazé et al.: Periods for extragalactic Of?p stars
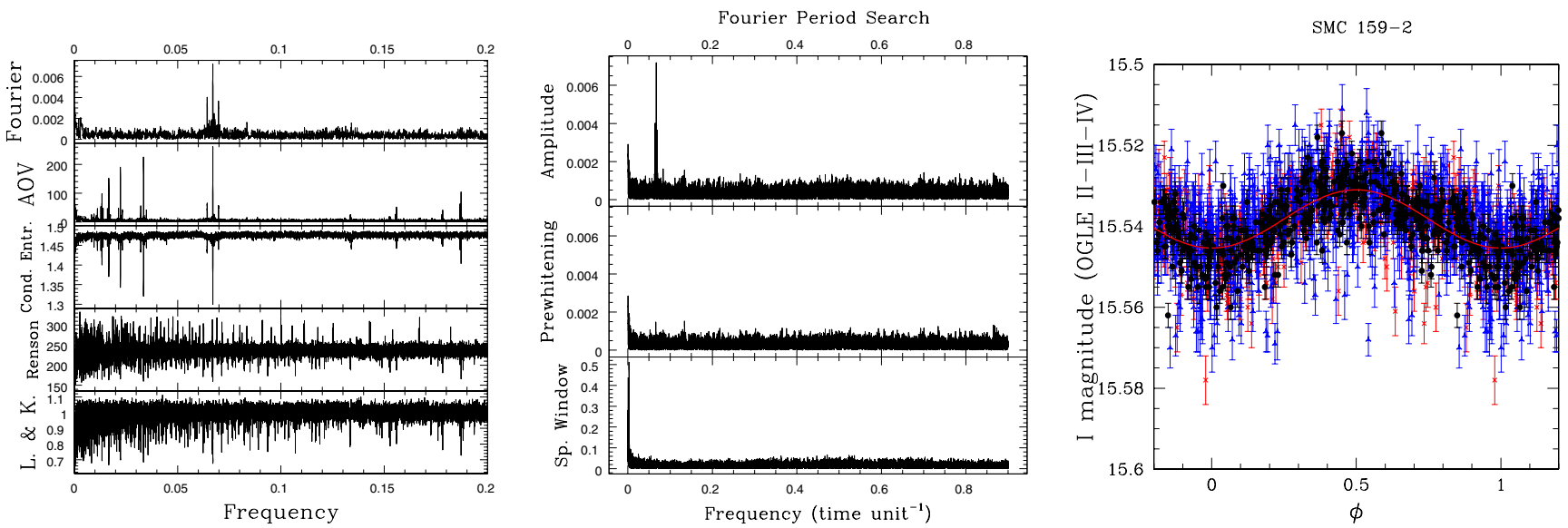

Fig. 8. Same as Fig. 5 for SMC159-2 (see Table 2 for ephemeris). 